\title{
Hydraulic technological machines with adaptive drive structure
}

\author{
Mikhail Lemeshko ${ }^{1, *}$, Mikhail Molev ${ }^{1}$, and Igor Golovin ${ }^{2}$ \\ ${ }^{1}$ Don state technical university (branch) In Shakhty Rostov region, Russia \\ ${ }^{2}$ Shakhty Polytechnic college, Shakhty, Rostov region, Russia
}

\begin{abstract}
In a large group of technological machines, drive provides two interlocking working movements. In metalworking, the working process is provided by two movements of the cutting tool relative to the forms or details. As a rule, this is due to the rotational and forward movements. The ratio of speeds of these movements, the ratio of two movements, or the ratio of the brought capacities to each of these movements can be the significant indicators of working process, having the optimum value. The method of automatic maintenance of the ratio for a hydraulic actuator with two engines is considered in the article. Schematic diagrams of the drive for some technological machines are considered. Based on the example of the device intended for processing of curvilinear surfaces, the process of automatic control regulating thickness of hydraulic actuator having adaptive structure is described. The efficiency of self-regulation at rather simple circuitry decisions is shown.
\end{abstract}

There are studies and developments devoted to the structures of adaptive mechanisms and adaptive machines [1,2]. In this case, the term "adaptive mechanism" is understood as a mechanism in which one movement is parametrically associated with another movement, as the interrelation of movements between each other. On this principle, mechanisms and machines are built to provide automatic self-tuning to the loads on each of the movements, providing rational relationship.

A great number of researches in the field of using adaptive mechanisms and adaptive machines were carried out by Professors A. Drovnikov and G.Vodyanik, and their students. For example, famous works $[3,4,5,6]$, reflecting the concept of adaptive machines based on two-differential systems.

They developed adaptive machines in which the optimization of operating modes under changing operating conditions is ensured by the machine structure, by means of using twodifferential connections between drives performing two working movements. The dynamics of such machines are well discussed in the work [7].

Let us consider some typical examples.

There is a device for stabilizing thickness of the removed layer for machining curvilinear surfaces [8]. The scheme of such device is shown in Figure 1.

\footnotetext{
${ }^{*}$ Corresponding author: lem-mikhail@ya.ru
} 


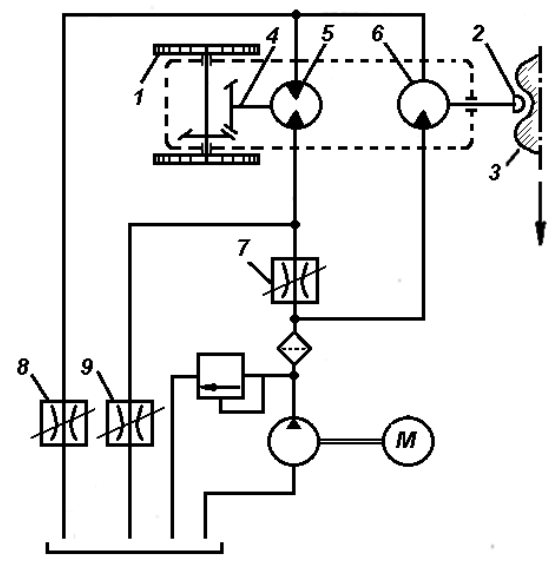

Fig. 1. Schematic diagram of the device for stabilization of thickness of the removed layer for machining curvilinear surfaces.

The principle of adaptation of this device is based on automatic change of the amount of feed rate, depending on the moment of resistance to rotation of a rotary type machining tool, for example, milling cutters.

The principle is used: when the thickness of the chips is increased (the cutting depth), the moment of rotation resistance is also increased, so, this increase in the automated hydraulic drives automatically reduces the feeding to the equilibrium state of the moment of resistance to rotation. When the cutting depth is reduced, the moment of resistance to rotation is also reduced, and the feeding rate is automatically increased. It should be noted that the automatic adjustment and stabilization of the removed layer is carried out through the force-moment connections. In the described device (Fig. 1), the feeding is carried out by moving the carriage 1 with the milling cutter 2, which leads to the approach or removal of the milling cutter 2 to the curved surface 3 being processed. Approach or removal of the carriage 1 is ensured by a reversible hydraulic motor 5 via the transmission 4 . When the curved surface 3 moves in the direction indicated by the arrow (see Fig. 1) the distance between the milling cutter 2 and the curved surface 3 will be changed. The chip thickness will be increased and the rotational resistance moment on the rotation motor 6 will be also increased. The pressure from the hydraulic pump through the throttle 7 will be increased, and the pressure at the output from the motor 6 will be decreased. The reversible motor will move the carriage to the left and the distance between the milling cutter 2 and the curved surface 3 is stabilized.

It is more convenient to analyze the described mechanism if we compose its structural scheme. In Fig. 2 the schematic diagram of the above device is given. For the convenience of the analysis and calculation of the structural diagram, a transition is done from an openloop hydraulic drive to a closed type of it when the drain lines are connected.

On the structural diagram it is convenient to explain why such schemes are called twodifferentiated. Indeed, the first hydraulic differential is formed by a hydraulic pump, a throttle 7 and a hydraulic motor 6. It is obviously expressed as $\mathrm{Q}_{h p}=\mathrm{Q}_{7}+\mathrm{Q}_{6}$. The second differential is hydro mechanical as it is formed by a reversible hydraulic motor, more precisely a rotation shaft and two hydraulic lines connected to a reversible hydraulic motor.

As a result of the above performed analysis, it should be noted that in the two-motor hydraulic drive under study, the machine adapts to a change in the curvature of the treated surface. In the future, the universality of the reduced structure will be shown, being implemented in many machines and devices based on so-called two-differential schemes. 


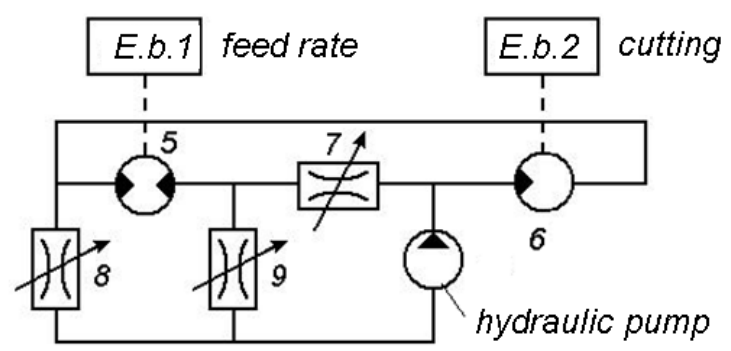

Fig. 2. Schematic diagram of the device for stabilizing the thickness of the removed layer of machining curvilinear surfaces (AS SU No. 483224).

Without dwelling on deeper calculations and analyzes, let us consider other machines having an adaptive structure.

Figure 3 shows a diagram of a hydraulic loading machine with adaptive qualities [9]. To analyze the adaptive properties, it is possible to represent the hydraulic drive of this machine in the form of a closed hydraulic drive with allocation of differential groups. Figure 4 shows the structure of this hydraulic drive that is identical to the diagram shown in Figure 3. In the actual loading machine, the hydraulic drive is not closed.

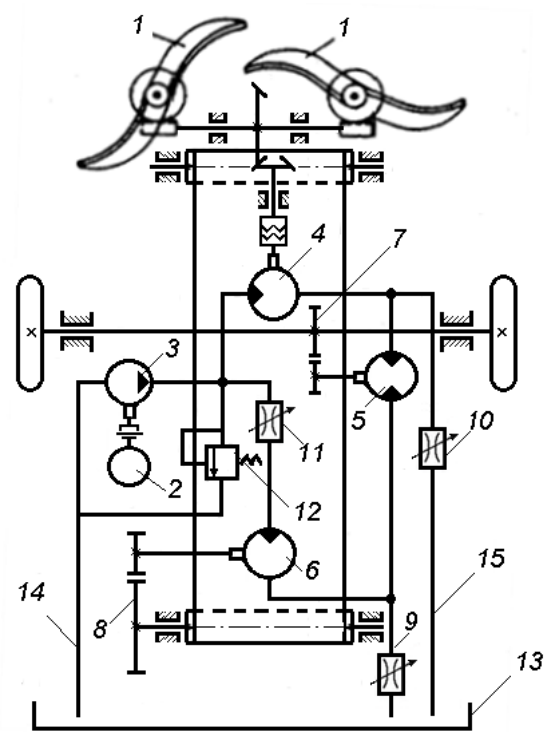

Fig. 3. Diagram of hydraulic loading machine.

1- loading body, 2 electric pump drive, 3-hydraulic pump, 4-engine loading unit, 5-reverse hydraulic motor, 6-motor conveyor, 7-feed reducer, 8-reducer conveyor, 9,10,11 -throttles

On the received scheme it is possible to allocate two hydraulic differentials. The first differential is the flow divider from the output of the hydraulic pump 3.It is obviously, the expenses of three elements of the circuit: 3,9,10 are expressed by the expression: $\mathrm{Q}_{3}=\mathrm{Q}_{11}$ $+\mathrm{Q}_{4}$. This is an important differential, because it binds the load on the drive of the Executive body 1( E.b. 1), and the load of the group of actuators 5 ( E.b. 2), 6 ( E.b. 3). The second differential is the pressure adder and expenses through the reversible hydraulic motor 5 .

Such a loading machine works as follows. The hydraulic fluid from the pump 3 is fed to the hydraulic motor 4 of the loading mechanism - (E.b.1) (thrusting paws). On the second hydraulic line from the pump through the throttle 11 , the hydraulic fluid enters the 
hydraulic motor 6 of the conveyor drive of the loading machine ( E.b.3) and then to the input of the reversible hydraulic motor 5 of the loading machine feeding onto the stack (E.b.2) of the feed material. The other input of this hydraulic motor is connected to the hydraulic line from the output of the hydraulic motor 4 , which is also connected to the drain line via the throttle 10 .

The working state of the hydraulic circuit is ensured by the main flow of the hydraulic fluid through the hydraulic motor 4 , the hydraulic motor 5 and the throttle 9 . The throttle 10 , adjusts the ratio of the loads of the hydraulic motors 4 and 5 . When the load on the hydraulic motor 4 is increased, the pressure at the inlet to the throttle 11 and then on the hydraulic motor 6 on the throttle 9 is also increased. At the same time, the speed of the reverse motor is decreased, the speed of the loading machine on the stack is also decreased, while the rotation speed of the conveyor drive is increased.

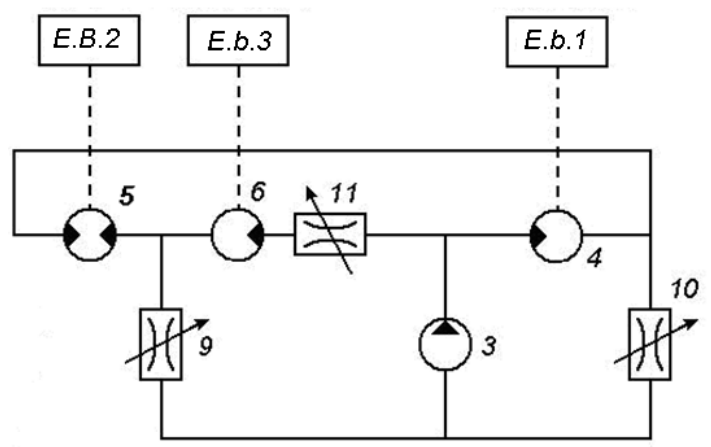

Fig. 4. Transformed (structural) scheme of the hydraulic drive of loading machine.

With the reduction of the load on the hydraulic motor 4, the thrust speed (motor rotation speed 5) of the loading machine on the stack is increased. The method is supposed to be a continuous loading process of the loading machine to the coalface. The thrust speed is adjustable and depends on the moment of resistance to the rotation of the drive of the paws.

So, the loading machine is known to be a self-regulated one. This class of machines with similar structures is called adaptive machines. Thus, the adaptability of the considered scheme is provided by two hydraulic differentials [10].

Let us consider the structural diagram of a drilling machine [11] with a special, adaptive drive (Fig. 5).

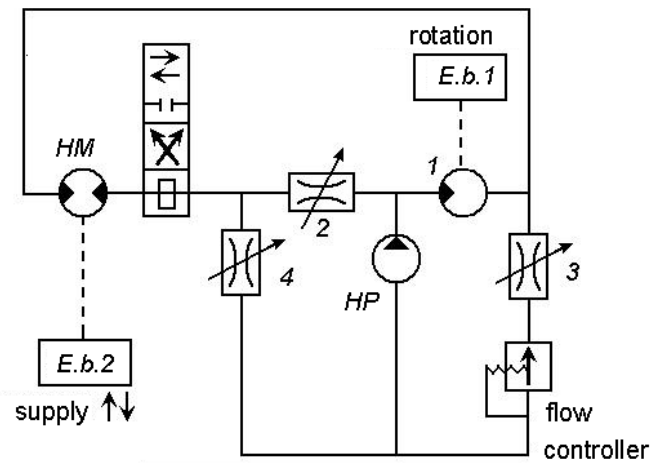

Fig. 5. Method of intensification of the cutting process during drilling. 1-motor of drill rotation; 2,3,4 - adjusting throttles 
In this machine, the feed rate is adjusted depending on the moment of resistance to rotation. Settings can provide maximum performance, within limits. Similarly to the above descriptions, the hydraulic drive of other adaptive machines and devices works. For example, the hydraulic drive of the terminal module of the robot [12], the hydraulic drive of the adaptive drilling machine [13].

Based on the analysis of structural circuits of well-known adaptive machines, we developed an adaptive drilling machine with hydro cylinder of feeding [13].

Hydraulic and structural diagrams of this machine are shown in Fig. 6, detailed description is given in papers $[13,14$.] When constructing the structural diagram, the hydro kinematic scheme of the adaptive machine was transformed into a circuit which is convenient for analysis, connecting the drain lines of all elements with the input to the hydraulic motor.
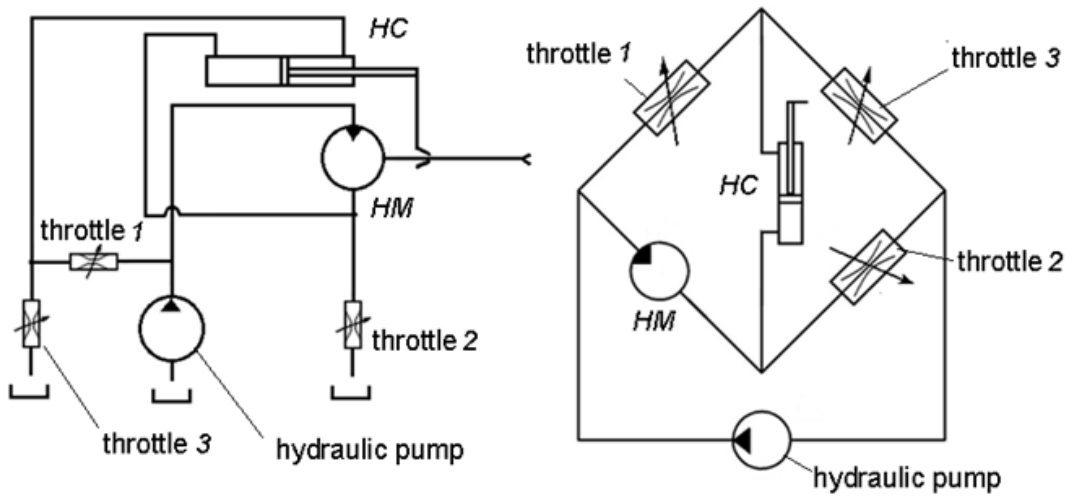

Fig. 6. Hydraulic and structural diagram of the developed drilling machine.

HP - hydraulic pump, HM - hydraulic motor, HC - hydraulic cylinder, Th1, Th2, Th3 - throttles settings of operating modes.

It is obvious, that these schemes are identical. It should be noted that the closed circuit of the drilling machine is a classic type of "bridge circuit" for hydro elements. This circumstance, in the future, is the basis for the calculation of hydraulic circuits having a similar structure.

The above described hydraulic drive: the drive of the device for stabilizing the thickness of the removed layer during machining of the curved surfaces of the drill, the drives of the drilling machine and the drilling machines can be represented by bridging switching on the hydraulic motor or hydraulic cylinder in the diagonal of the bridge.

It is obvious, that depending on the ratio of the bridge arms, the flow direction to the diagonal can be direct or reverse.

Analysis of adaptive hydraulic and hydro-mechanical circuits showed that there is a unity between these schemes, that can be represented as a classical bridge circuit.

The design and calculation of a hydraulic drive having an adaptive structure can be simplified by applying an electro hydraulic analogy [15], where the throttles, hydraulic cylinder, hydraulic motor can be represented as resistances in the bridge electrical circuit, the analogy of the current in the circuit is the flow rate of the hydraulic fluid of this circuit, pressure and voltage.

Bridge circuits in electrical engineering can be easily estimated by various known methods, for example, the method of loop currents 


\section{References}

1. G.M. Vodianik, A.N. Drovnikov, I.P. Streltsov, Mining equipment and electromechanics. № 10. pp. $54-57$ (2007)

2. A.N. Drovnikov, Theory and practice of adaptive mechanisms, Part 1, part 2. / News. polytech. in-t - Novocherkassk., Dep. in the VINITI 5.12.83., $36570-83 \mathrm{D}$ and 6571 83D, 445 p. (1983)

3. A.N. Drovnikov, G.D. Dibrova, Structurally-adaptive technical systems / Monograph / A.N. Drovnikov, G.D. Dibrova; Ministry of Education and Science of the Russian Federation, Federal State. budget educational institution. "South-Russian State University of Economics and Service". Shakhty, p. 67., (2011)

4. G.M. Vodyanik, E.V. Rileev, A.N. Drovnikov. Research and development of a drilling machine with automatic feed rate control for drilling vertical holes up to $2.5 \mathrm{~m}$. Report. Work No. 1340, part 11, 111, Novocherkassk, NPI., 266 p (1968)

5. G.M. Vodianik, A.N. Drovnikov, V.S. Krutikov, A.I. Butov, V.M. Bocharov, M.G.Vodyanik, V.L. Kondrashev, Electromachine and hydraulic machine differentials in the adjustable drive of mining machines, In the collection: Scientific and pedagogical schools (NPI). History. Achievements. Contribution to domestic science. Collection of scientific articles. Editor: VE Shukshunov. Novocherkassk,. pp. 96103(2007)

6. A.S. Kuznetsov, Investigation of two differential drive of a drilling machine with automatic feeding of BMVA-1 in production conditions. Diss.of Cand. tech. Sciences., NPI, 169 p. (1976)

7. A.N. Drovnikov, M.A. Lemeshko Structural diagram of the dynamic model "adaptive drilling rig - slaughter" / Mining information analytical bulletin (scientific and technical journal).. № 8. pp. 147-149 (2003)

8. A.c. SU № 483224 IPC B23Q5 / 06, B24b5 / 16. Published: 09/05/1975. A device for stabilizing the thickness of the layer to be removed during machining of curvilinear surfaces / Shakhtinsky Technological Institute of Consumer Services // A.N.Drovnikov, G.M. Vodyanik, V.A. Pershin (1975)

9. A.c USSR №1126701. Published. 22.07.83. Hydraulic drive of the loading machine / Shakhtinsky Technological Institute of Consumer Services // V.A. Boltovsky, Yu.E. Karandeev, A.N. Drovnikov, N.F. Tsygankov (1983)

10. G.M. Vodyanik, A.N. Drovnikov, Yu. A. Vasiliev, Izvestiya of the North-Caucasian Scientific Center of Higher Education. Technical science, № 1, pp.123-126 (1973)

11. A.c. USSR № 929331 Published on 04.07. 1979. A method for intensifying the cutting process / V.I. Tolubets, V.A. Pershin, V.V. Gvozdev, L.V. Larina, A.N. Drovnikov (1979)

12. A. c USSR No. 1318392. The terminal module of the robot, A.N. Drovnikov, I.V. Darda (1975)

13. Patent RUS No. 2473767. Published on 07/29/2011. Adaptive rotary drilling machine / M.A. Lemeshko, A.V. Trifonov (2011)

14. M.A. Lemeshko, A.V. Trifonov, Mining information analytical bulletin (scientific and technical journal), № 2, pp. 207-210 (2012)

15. M.A. Lemeshko, R.Yu. Volkov, Technological and technological problems of service.. No. 3 (29). pp. 62-65 (2014) 